\title{
ON NON-LINEAR ELLIPTIC EQUATIONS AND THE STABILITY OF SOAP FILM
}

\section{CôNg Nghê TRUong}

We use an Inverse Mapping Theorem for Sobolev chains to obtain a number of existence theorems for non-linear elliptic partial differential equations with general order. We then apply one of these theorems to prove the stability of a soap film.

\section{INTRODUCTION}

In this paper smoothness shall always mean $C^{\infty}$-smooth. For simplicity of exposition, our partial differential equations will have real coefficients and be defined on an open set of an Euclidean space $\mathbf{R}^{n}$ rather than on a manifold.

Let $\Omega$ be an open, convex, bounded subset of $\mathbf{R}^{n}$ with boundary $\partial \Omega$, a $C^{\infty}$. manifold, and let $\bar{\Omega}$ be its closure. Let $m \geqslant 1$ be an integer and $f_{0}, g$ be two $C^{\infty}$ functions on $\bar{\Omega}$. We are interested in finding $C^{\infty}$-solutions $u(x)$ of the non-linear elliptic partial differential equation:

$$
F\left(x, u(x), D u(x), \ldots, D^{2 m} u(x)\right)=g(x)
$$

which satisfy the Dirichlet boundary condition:

$$
u(x)=f_{0}(x) \text { for all } x \in \partial \Omega,
$$

where $F: J^{2 m}(\bar{\Omega}) \rightarrow \mathbf{R}$ is a $C^{\infty}$-map $[14]$.

One of the main methods used to study non-linear elliptic boundary problems is the topological method based on the degree of non-linear mappings. Another method for treating non-linear partial differential equations is the direct method of the calculus of variations. A very successful method for solving non-linear boundary problems is that of using a priori estimates of the solutions. Yet another method uses non-linear operators of monotone type. For details of these methods, see Agmon, Douglas and Nirenberg [1], Bers, John and Schechter [3], Browder [5], Courant and Hilbert [8], Garabedian [10] and Nirenberg [13]. The natural settings for all these methods are Banach (or Hilbert) spaces where the inverse Mapping Theorem holds.

Received 11th August 1997.

Copyright Clearance Centre, Inc. Serial-fee code: 0004-9729/98 \$A2.00+0.00. 
Unfortunately, it has been known for several decades that, for many problems in non-linear analysis, Banach spaces and Banach manifolds are inadequate. For instance, they are not suitable for applications to the Cauchy problem of an equation of evolution, and to the space of $C^{\infty}$-diffeomorphisims on a compact manifold. Marsden's note [12] is a good survey of these and other related matters. In fact, it has long been recognised that, for these problems and for our boundary problem above, the spaces involved are the more general Frechet spaces where the Inverse Mapping Theorem is no longer valid. A counter-example was given by Eels [9] which showed that the Inverse Mapping Theorem is false for separable Frechet spaces endowed with any reasonable traditional differentiation in the norms.

In 1975, in an attempt to overcome this difficulty, Yamamuro [20] developed a differentiation in (non-normed) locally convex spaces for which the Inverse Mapping Theorem holds. He terms it B $\Gamma$-differentiation. At about the same time, Omori [14], in working towards a theory of general Lie groups, introduced Inverse Limit Banach (ILB) and Inverse Limit Hilbert (ILH) Lie groups and proved an Inverse Mapping Theorem for them. Together with Hamilton's version of the Nash-Moser Inverse Mapping Theorem, Omori's method is the most fruitful, but it is not very accessible and is of somewhat ad hoc nature.

In [18], the author of the present paper generalised Yamamuro's differentiation to give an axiomatic setting for the method of Omori and obtain an Inverse Mapping Theorem for Sobolev chains. The main idea is to save as much Banach space technique as possible in the realm of those locally convex spaces that model manifolds of mappings. It turns out that this Inverse Mapping Theorem can be exploited to obtain a number of results on the existence and uniqueness of the solutions of boundary value problems for a general non-linear elliptic partial differential equation with smooth real coefficients, results which will be given in this paper in Theorems I, II III and IV.

An outstanding example of a non-linear elliptic Dirichlet boundary problem is Plateau's problem of finding a minimal surface spanned inside a given contour. Closely related to Plateau's problem is the soap film problem: When a closed contour made of wire is dipped into a soap film solution, a thin film of soap is formed within the wire. So far in the literature, by ignoring the difference in pressure on the two surfaces of the soap film, mathematicians have treated a soap film as a minimal surface and proved its existence (see, for example, Garabedian [10], Courant [7]). The stability of a soap film has, however, been neglected. Fortunately, using Theorem I, we shall show that a soap film, if it exists, will be stable.

The paper consists of three sections. In Section 1 we present our main theorems. Section 2 is for the proofs of the main theorems. The final section, Section 3 , is for application to the stability of the equilibrium of a soap film. 


\section{The MaIN Theorems}

Let $\Omega$ be an open, convex, bounded subset in $\mathbf{R}^{n}$ with boundary $\partial \bar{\Omega}$, a smooth manifold. Denote by $C^{\infty}(\bar{\Omega})$ the space of all $C^{\infty}$-maps from $\bar{\Omega}$ into $\mathbf{R}$. Then $C^{\infty}(\bar{\Omega})$ is a Sobolev chain [18] endowed with a sequence of Banach-norms (or Hilbert-norms) as defined in Omori $\left[14\right.$, p.28]. Let $m$ be an integer $\geqslant 1$, let $g$ be an element of $C^{\infty}(\Omega)$ and consider the following non-linear elliptic partial differential equation of order $2 m$ with smooth real coefficients:

$$
F\left(x, u(x), D u(x), \ldots, D^{2 m} u(x)\right)=g(x)
$$

where $F: J^{2 m}(\bar{\Omega}) \rightarrow \mathbf{R}$ is a $C^{\infty}$-map.

We are interested in finding $C^{\infty}$-solutions which satisfy the Dirichlet boundary condition

$$
u(x)=f_{0}(x) \text { for all } x \in \partial \Omega
$$

where $f_{0}$ is in $C^{\infty}(\bar{\Omega})$.

Denote by $C_{f_{0}}^{\infty}(\bar{\Omega})$ the affine subspace of $C^{\infty}(\bar{\Omega})$ consisting of maps which agree with $f_{0}$ on $\partial \bar{\Omega}$ and define:

$$
\Phi: C_{f_{0}}^{\infty}(\bar{\Omega}) \rightarrow C^{\infty}(\bar{\Omega})
$$

by:

$$
\Phi(u)(x)=F\left(x, u(x), D u(x), \ldots, D^{2 m} u(x)\right)
$$

Then (1) can be written as:

$$
\Phi(u)=g
$$

For each $u$ in $C_{f_{0}}^{\infty}(\bar{\Omega})$, consider the linearised equation of (4):

$$
A_{u}(\eta)=\zeta \text { for } \zeta \in C^{\infty}(\bar{\Omega})
$$

where $\eta \in C^{\infty}(\Omega)$ and

$$
A_{u}=\sum_{|\alpha| \leqslant 2 m} \frac{\partial F}{\partial D^{\alpha} u}\left(x, u(x), D u(x), \ldots, D^{2 m} u(x)\right) D^{\alpha} \eta
$$

Our main theorems are as follows. 
TheOREM I. Suppose that:

(i) For a given $g_{\circ}$ in $C^{\infty}(\bar{\Omega})$, the nonlinear equation $\Phi(u)=g_{0}$ has a solution $u_{0}$ in $C_{f_{0}}^{\infty}(\Omega)$;

(ii) The linearised equation $A_{u_{o}}(\eta)=0$ has $\eta=0$ as the only solution in $C_{0}^{\infty}(\bar{\Omega})$.

Then, for every $g$ sufficiently near $g_{o}$ in $C^{\infty}(\bar{\Omega})$, the non-linear equation $\Phi(u)=g$ has one and only one solution $u$ near $u_{0}$ in $C_{f_{0}}^{\infty}(\bar{\Omega})$. Moreover, the solution $u$ depends smoothly on $g$.

THEOREM II. Suppose that

(i) For a given $g_{0}$ in $C^{\infty}(\bar{\Omega})$, the non-linear equation $\Phi(u)=g_{0}$ has a solution $u_{0}$ in $C_{f_{0}}^{\infty}(\bar{\Omega})$;

(ii) For all $\zeta$ in $C^{\infty}(\bar{\Omega})$, the linearised equation $A_{u_{0}}(\eta)=\zeta$ has a solution in $C_{0}^{\infty}(\bar{\Omega})$.

Then for all $g$ sufficiently near $g_{0}$ in $C^{\infty}(\bar{\Omega})$, the non-linear equation $\Phi(u)=g$ has a unique solution $u$ near $u_{0}$ in $C_{f_{0}}^{\infty}(\bar{\Omega})$. Moreover, the solution $u$ depends smoothly on $g$.

Theorems I and II are perturbation theorems similar to the ones given by Courant and Hilbert [8, p.368] and Agmon, Douglas and Nirenberg [1, p.690], which are both obtained using Schauder estimates. Our Theorems I and II are, however, stronger in the sense that the unique solution $u$ depends smoothly on the right-hand-side $g$. This strength will be used to prove the stability of the equilibrium of a soap film in Section 3. It should not be too surprising that our Inverse Mapping Theorem has led to a result similar to that of [1, Theorem 12.6], since this Inverse Mapping Theorem was based on Garding inequalities which are themselves a a priori estimates.

THEOREM III. For almost all $g$ in $C^{\infty}(\bar{\Omega})$ (that is, except on a set of first category) the solution space of the non-linear equation $\Phi(u)=g$ is discrete. In other words, for almost all $g$ in $C^{\infty}(\bar{\Omega})$, solutions of the equation $\Phi(u)=g$ are locally unique.

This Theorem III is a $C^{\infty}$-version of a result given by Smale [15]. It is proved using a $\beta \Gamma$-version of the Smale Density Theorem which is also a direct consequence of the Inverse Mapping Theorem in [18]. It should be noted that this Inverse Mapping Theorem has been used in [18] to prove the finite-dimensionality of the solution of $\Phi(u)=g$ for all $g$ in $C^{\infty}(\Omega)$, a result of Sunada [16].

THEOREM IV. Suppose that

(i) For all $u$ in $C_{f_{0}}^{\infty}(\Omega)$ the linearised equation $A_{u}(\eta)=0$ has $\eta=0$ as the only solution in $C_{0}^{\infty}(\Omega)$; 
(ii) For each $g_{0}$ in $C^{\infty}(\bar{\Omega})$ and for each $i \geqslant n+6$, there exist constants $k_{i}\left(g_{0}\right)>0, \varepsilon_{i}\left(g_{0}\right)>0$ such that if $u$ is in $C_{f_{0}}^{\infty}(\bar{\Omega}), \Phi(u)=g$ and $\left\|g-g_{0}\right\|_{i}<\varepsilon_{i}$, then $\|u\|_{i+2 m}<k_{i}$, where $\|\cdot\|_{i}$ is the $H$-norm defined as above.

Then, for each $g$ in $C^{\infty}(\bar{\Omega})$, the non-linear equation $\Phi(u)=g$ has one and only one solution $u$ in $C_{f_{0}}^{\infty}(\Omega)$.

Theorem IV is an existence and uniqueness theorem which is a $C^{\infty}$-version of a theorem of Browder [4, Theorem 3].

\section{Proofs of the Main Theorems}

The proofs of the main theorems are based on the Inverse Mapping Theorem in [18]. First let us extend the $C_{\beta \Gamma}^{1}$-maps in [18] to $C_{\beta \Gamma}^{k}$-maps for $k \geqslant 2$ or $\infty$.

Let $\left\{E, E^{i}: i \geqslant d\right\}$ and $\left\{F, F^{i}: i \geqslant d\right\}$ be two Sobolev chains [18], let $U \subseteq E$ be open and $f: U \subseteq E \rightarrow F$ be a map and $k$ be an integer $\geqslant 2$.

DEFinition 2.1: We say the $f$ is of class $C_{\beta \Gamma}^{k}$ if and only if:

(i) The map $f: U \subseteq E \rightarrow F$ is of class $C_{\beta \Gamma}^{1}$ as defined in [18]; and

(ii) The derived map $D f: U \subseteq E \rightarrow L_{\beta \Gamma}(E, F)$ is of class $C_{\widehat{\Gamma}}^{k-1}$ with respect to the calibration $\widehat{\Gamma}=\left\{\left(|.|_{i},\|,\|_{i}\right): i \geqslant d\right\}$ for $\left(E, L_{\beta \Gamma}(E, F)\right)$ as defined in [18, p.384].

$f$ is said to be of class $C_{\beta \Gamma}^{\infty}$ if it is of class $C_{\beta \Gamma}^{k}$ for all $k \geqslant 1$.

We define $\beta \Gamma$-splitting linear maps and $\beta \Gamma$-Fredholm linear maps exactly as in [19] replacing $B \Gamma$ by $\beta \Gamma$.

Definition 2.2: A map $f: U \rightarrow F$ is called a $\beta \Gamma$-Fredholm map of class $C_{\beta \Gamma}^{k}$ if it is a $C_{\beta \Gamma}^{k}$-map on $U$ and, for each $a \in U, f^{\prime}(a)$ is a $\beta \Gamma$-Fredholm linear map. For a $\beta \Gamma$-Fredholm map $f: U \rightarrow F$ of class $C_{\beta \Gamma}^{k}$, the set of all $a \in U$ such that $f^{\prime}(a)$ is not surjective is called the critical set of $f$ and is denoted by $C(f, U)$.

We are now ready to prove the main theorems. By a translation, we may assume that $f_{0}=0$. Then: $\Phi: C_{0}^{\infty}(\bar{\Omega}) \rightarrow C^{\infty}(\bar{\Omega})$ where $C_{0}^{\infty}(\bar{\Omega})$ and $C^{\infty}(\bar{\Omega})$ are Sobolev chains [18]. The following lemma plays an essential role in the proofs of our main theorems. It follows from standard results in Bers, John and Schechter,[3, Part II, Chapter 5, p.199] and [18]. See also Hormander [11], Smale [15] and Browder [4].

LEMMA 2.3. $\Phi: C_{0}^{\infty}(\bar{\Omega}) \rightarrow C^{\infty}(\bar{\Omega})$ is a regular $C_{\beta \Gamma}^{1}$ map if we endow $\left(C_{0}^{\infty}(\bar{\Omega})\right.$, $\left.C^{\infty}(\Omega)\right)$ with the calibration $\Gamma=\left\{\left(\|\cdot\|_{t+2 m},\|\cdot\|_{i}\right): i \geqslant n+6\right\}$ as defined in [18]. The 
$\beta \Gamma$-derivative of $\Phi$ at $u \in C_{0}^{\infty}(\bar{\Omega})$ is:

$$
D \Phi(u) \cdot \eta=\sum_{i=0}^{2 m} \frac{\partial F}{\partial D u^{i}} D^{i} \eta(x)
$$

where $\eta \in C_{0}^{\infty}(\bar{\Omega})$.

Furthermore, if $F$ is elliptic then $D \Phi(u)$ is a $\beta \Gamma$-Fredholm operator with index 0 .

By (i), (ii) and Lemma 2.3, $\Phi$ is a regular $C_{\beta \Gamma}^{1}$-map at $u_{0} \in C_{0}^{\infty}(\bar{\Omega})$ and $D \Phi\left(u_{0}\right) \epsilon$ $G L_{\beta \Gamma}\left(C_{0}^{\infty}(\bar{\Omega}), C^{\infty}(\bar{\Omega})\right)$. Thus Theorem I follows.

Similarly since the index of $A_{u_{0}}$ is $0, \Phi$ is a regular $C_{\beta \Gamma}^{1}$-map and $u_{0}$ and $D \Phi\left(u_{0}\right) \epsilon$ $G L_{\beta \Gamma}\left(C_{0}^{\infty}(\Omega), C^{\infty}(\bar{\Omega})\right)$. Thus Theorem II follows.

In view of Lemma 2.3, Theorem III follows from the following result whose proof is similar to that of Smale [15] in the case of Banach spaces.

Propos It Ion 2.4. Let $\mathfrak{F}$ be a $\Gamma$-family of Sobolev chains. Let $E, F \in \mathfrak{F}$ and let $U$ be an open subset of $E$. Let $f: U \rightarrow F$ be a $\beta \Gamma$-Fredholm map of class $C_{\beta \Gamma}^{k}$ such that $k>\max (\operatorname{index}(u), 0)$ and $f^{\prime}(a)=u$ for some $a \in U$. Then there exists an open neighbourhood $W$ of a such that $f[C(f, U) \cap W]$ is nowhere dense and closed.

Finally, since $\Phi$ is a regular $C_{\beta}^{1}$-map, for each $i \geqslant d, \Phi$ extends to a $C^{1}$-map $\widetilde{\Phi}: H_{0}^{i+2}(\bar{\Omega}) \rightarrow H^{i}(\bar{\Omega})$ between Hilbert spaces where $H_{0}^{i+2}(\bar{\Omega})$ (respectively $H^{i}(\bar{\Omega})$ ) is the completion of $C_{0}^{\infty}(\bar{\Omega})$ (respectively $C^{\infty}(\bar{\Omega})$ ) with respect to the norm $\|\cdot\|_{i+2}$ (respectively $\|\cdot\|_{i}$ ). It then follows that $\widetilde{\Phi}$ is a homeomorphism of $H_{0}^{i+2}(\bar{\Omega})$ onto $H^{i}(\bar{\Omega})$ for all $i \geqslant d$. Therefore $\Phi: C_{0}^{\infty}(\bar{\Omega}) \rightarrow C^{\infty}(\bar{\Omega})$ is a homeomorphism onto. Thus Theorem IV follows.

\section{STABILITY OF THE EQUILIBRIUM OF A SOAP FILM}

In [2] the equilibrium of a soap film is discussed on the hypothesis that there is a certain type of surface energy of mechanical type associated with each element of the surface. This energy, called the tension-energy, is represented by the integral $\iint T d s$ taken over the portion of surface under consideration. $T$ is a constant, called the surface tension which is not dependent on the shape and size of the film but it does depend upon the temperature. A soap film is considered as having two surfaces which are endowed with tension-energy [2].

When a film attached to a wire is in equilibrium under the forces of tension alone, the total tension-energy is a minimum. Assuming that the $z$-coordinate of a point on the surface or rim of the film is a function of $x$ and $y$, then the total tension-energy $E$ is:

$$
E=2 T \iint\left(1+z_{x}^{2}+z_{y}^{2}\right)^{1 / 2} d x d y
$$


Hence the Eulerian equation of the calculus of variations gives

$$
\frac{\partial}{\partial x}\left(\frac{z_{x}}{H}\right)+\frac{\partial}{\partial y}\left(\frac{z_{y}}{H}\right)=0
$$

where

$$
H=\left(1+z_{x}^{2}+z_{y}^{2}\right)^{1 / 2} .
$$

This is the differential equation of a minimal surface.

When the film is subjected to a difference of pressure on the two sides and the fluid on one side of the film is a closed vessel whose pressure is $p_{1}$ while the pressure on the other side of the film is $p_{2}$, there is a pressure energy $\left(p_{1}-p_{2}\right) V$ associated with the vessel closed by the film, where $V$ is the volume of this vessel (see [2]). Write

$$
V=V_{0}+\frac{1}{3} \int \omega d s
$$

where $V_{0}$ is a constant and $\omega$ is the perpendicular from the origin to the surface element $d s$ and consider the variation of the integral

$$
\iint H\left[2 T+\frac{1}{3} \omega\left(p_{1}-p_{2}\right)\right] d x d y
$$

Since $\omega H=z-x z_{x}-y z_{y}$ the differential equation for the soap film is:

$$
p_{1}-p_{2}+2 T\left[\frac{\partial}{\partial x}\left(\frac{z_{x}}{H}\right)+\frac{\partial}{\partial y}\left(\frac{z_{y}}{H}\right)\right]=0
$$

or, equivalently,

$$
\frac{\partial}{\partial x}\left(\frac{z_{x}}{H}\right)+\frac{\partial}{\partial y}\left(\frac{z_{y}}{H}\right)=p
$$

where

$$
p=\frac{p_{2}-p_{1}}{T}
$$

It is easy to see that (7) and (9) reduce to non-linear elliptic partial differential equations:

$$
\left(1+z_{y}^{2}\right) z_{x x}-2 z_{x} z_{y} z_{x y}+\left(1+z_{x}^{2}\right) z_{y y}=0
$$

and

$$
\left(1+z_{y}^{2}\right) z_{x x}-2 z_{x} z_{y} z_{x y}+\left(1+z_{x}^{2}\right) z_{y y}=p
$$


A solution of $\left(7^{\prime}\right)$ represents a minimal surface whereas a solution of $\left(9^{\prime}\right)$ represents a soap film. Note that when the difference of pressure on the two sides of a soap film is 0 a soap film becomes a minimal surface. In the literature, mathematicians assumed no pressure difference and treated a soap film as a minimal surface when proving the existence of a soap film (see for example, $[7,9]$ ). Soap film experiments have shown that the equilibrium of a soap film is stable [6]. Mathematically, a soap film $z(x, y)$ is stable if, whenever the difference-pressure $p$ varies a little, $z(x, y)$ varies a little. In other words, if $z_{0}(x, y)$ is a solution of $\left(9^{\prime}\right)$ corresponding $p=p_{0}$ then there exists $\varepsilon>0$ such that for all $p^{*}$ such that $\left|p^{*}-p_{0}\right|<\varepsilon$ the equation

$$
\left(1+z_{y}^{2}\right) z_{x x}-2 z_{x} z_{y} z_{x y}+\left(1+z_{x}^{2}\right) z_{y y}=p^{*}
$$

has a solution $z^{*}(x, y)$ close to $z(x, y)$.

Now using Theorem I and the results in [3], it is easy to prove the following.

Proposition 3.1. Let $C$ be a closed contour in $\mathbf{R}^{3}$ whose projection on a plane is a smooth curve enclosing a convex domain. Then a soap film spanning $C$, if it exists, is stable. Moreover, it depends smoothly on the pressure-difference.

When a soap film is subjected to only a small difference of pressure and is stretched across a thin flat contour we can, to a sufficient approximation, put $H=1$ in $\left(9^{\prime}\right)$. The resulting equation is:

$$
\frac{\partial^{2} \dot{z}}{\partial x^{2}}+\frac{\partial^{2} z}{\partial y^{2}}=k
$$

where $k$ is a constant and the boundary condition is $z=0$ on the rim. In this case the existence of a soap film is ensured and we have

Proposition 3.2. If $C$ is a thin flat smooth contour in $\mathbf{R}^{3}$ then there always exists a soap film spanning $C$. Moreover, the soap film is stable and depends smoothly on the pressure-difference.

\section{REFERENCES}

[1] S. Agmon, A. Douglas and L. Nirenberg, 'Estimates near the boundary for solutions of elliptic partial differential equations satisfying general boundary conditions', Comm. Pure Appl. Math. XII (1959), 623-727.

[2] H. Bateman, Partial differential equations of mathematical physics (Cambridge University Press, Cambridge, England, 1959).

[3] L. Bers and M. Schechter, Partial differential equations, Lectures in Applied Mathematics III (John Wiley and Sons, New York, 1964).

[4] F.E. Browder, 'Topological methods for non-linear elliptic equations of arbitrary order', Pacific J. Math. 17 (1966), 17-31. 
[5] F.E. Browder, 'Existence theorems for nonlinear partial differential equations', in Global Analysis, Proceedings of Symposia in Pure Mathematics XVI (American Mathematical Society, Providence, R.I., 1970).

[6] R. Courant, 'Soap film experiments with minimal surfaces', Amer. Math. Monthly 47 (1940), 167-174.

[7] R. Courant, Dirichlet's principle, conformal mapping and minimal surfaces (Interscience, New York, 1950).

[8] R. Courant and D. Hilbert, Methods of Mathematical Physics 2 (Interscience, New York, 1961).

[9] J. Eels, 'A setting for global analysis', Bull. Amer. Math. Soc. 72 (1966), 751-807.

[10] P.R. Garabedian, Partial differential equations (John Wiley and Sons, New York, 1964).

[11] L. Hormander, Linear partial differential operators, (Third revised printing) (SpringerVerlag, Berlin, Heidelberg, New York, 1969).

[12] J.E. Marsden, Hamiltonian mechanics, infinite dimensional Lie groups, Geodesic flows and hydrodynamics, (Notes at Berkeley, California, 1969).

[13] L. Nirenberg, 'On elliptic partial differential equations', Ann. Scuola Norm. Sup. Pisa Cl. Sci. 13 (1969), 115-162.

[14] H. Omori, Infinite dimensional Lie transformation groups, Lecture Notes in Mathematics 427 (Springer-Verlag, Berlin, Heidelberg, New York, 1974).

[15] S. Smale, 'An infinite dimensional version of Sard's Theorem', Amer. J. Math. 87 (1965), 861-866.

[16] T. Sunada, 'Non-linear elliptic operators on a compact manifold and an implicit function theorem', Nagoya Math. J. 57 (1974), 175-200.

[17] C.N. Truong, 'Manifolds of smooth maps', Bull. Austral. Math. Soc. 24 (1981), 1-11.

[18] C.N. Truong, 'An inverse mapping theorem for Sobolev chains and its application', Bull. Austral. Math. Soc. 27 (1983), 281-394.

[19] C.N. Truong and S. Yamamuro, 'Locally convex spaces, differentiation and manifolds', Comment. Math. Special Issue 2 (1979), 229-338.

[20] S. Yamamuro, A theory of differentiation in locally convex spaces, Memoirs of the Americal Mathematical Society 212 (American Mathematical Society, Providence, RI, 1979).

School of Quantitative Business Methods and Operations

University of Western Sydney, Nepean

PO Box 10

Kingswood NSW 2747

Australia 